\title{
Artefact
}

Techniques, histoire et sciences humaines

\section{Nicolas Minvielle Larousse, Marie-Christine Bailly- Maître et Giovanna Bianchi (dir.), Les Métaux précieux en Méditerranée médiévale : exploitations, transformations, circulations}

Aix-en-Provence, Presses universitaires de Provence (Bibliothèque d'archéologie méditerranéenne et africaine, 27), 2019

\section{Luc Bourgeois}

\section{(2) OpenEdition}

Édition électronique

URL : http://journals.openedition.org/artefact/6112

DOI : 10.4000/artefact.6112

ISSN : 2606-9245

Éditeur :

Association Artefact. Techniques histoire et sciences humaines, Presses universitaires du Midi

Édition imprimée

Date de publication : 15 juillet 2020

Pagination : 355-358

ISBN : 978-2-8107-0691-4

ISSN : 2273-0753

Référence électronique

Luc Bourgeois, « Nicolas Minvielle Larousse, Marie-Christine Bailly-Maître et Giovanna Bianchi (dir.), Les Métaux précieux en Méditerranée médiévale : exploitations, transformations, circulations », Artefact [En ligne], 12 | 2020, mis en ligne le 21 décembre 2020, consulté le 23 décembre 2020. URL : http:// journals.openedition.org/artefact/6112; DOI : https://doi.org/10.4000/artefact.6112

Ce document a été généré automatiquement le 23 décembre 2020.

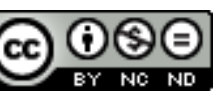

Artefact, Techniques, histoire et sciences humaines est mise à disposition selon les termes de la Licence Creative Commons Attribution - Pas d'Utilisation Commerciale - Pas de Modification 4.0 International. 


\section{Nicolas Minvielle Larousse, Marie- Christine Bailly-Maître et Giovanna Bianchi (dir.), Les Métaux précieux en Méditerranée médiévale : exploitations, transformations, circulations}

Aix-en-Provence, Presses universitaires de Provence (Bibliothèque d'archéologie méditerranéenne et africaine, 27), 2019

\section{Luc Bourgeois}

\section{RÉFÉRENCE}

Nicolas Minvielle Larousse, Marie-Christine Bailly-Maître et Giovanna Bianchi (dir.), Les Métaux précieux en Méditerranée médiévale : exploitations, transformations, circulations, Aixen-Provence, Presses universitaires de Provence (Bibliothèque d'archéologie méditerranéenne et africaine, 27), 2019, 339 pages.

1 Issus d'un colloque organisé à Aix-en-Provence du 6 au 8 octobre 2016, ces actes réunissent vingt-six contributions articulées en trois thèmes: la caractérisation des espaces miniers, les techniques et l'organisation de la production, la circulation et les usages des métaux précieux. L'impact environnemental des mines, axe de recherche dont l'importance récente est rappelée en introduction, n'a pas donné lieu à des développements particuliers.

2 L'argent domine très largement un recueil où l'or apparaît - en premier lieu celui extrait du continent africain (Julie Marchand et al. pour l'Égypte islamique) - surtout dans la section consacrée à la circulation des métaux. On peut penser que cette discrétion résulte à la fois de la difficulté à repérer les exploitations d'or alluvionnaire et de la domination de l'argent dans la production monétaire médiévale. La fréquence 
des gisements polymétalliques oblige également à évoquer fréquemment le plomb, le cuivre, le zinc, voire le fer. Dans l'ensemble du volume, le cuivre est d'ailleurs placé au rang des métaux précieux. La relative rareté des alliages cuivreux dans une large portion de l'Occident des $\mathrm{Ix}^{\mathrm{e}}$-XII ${ }^{\mathrm{e}}$ siècles plaide en effet pour cette interprétation, mais elle se justifie sans doute moins pour la fin de la période médiévale. Notons d'ailleurs que les derniers siècles du Moyen Âge occupent ici une place majeure, ce qui témoigne sans nul doute d'un essor de l'activité minière à cette époque mais peut également résulter pour partie de l'augmentation de la documentation textuelle et de l'occultation partielle des travaux antérieurs dans de nombreux districts miniers.

3 La première partie montre que l'étude des espaces miniers médiévaux a largement progressé dans le sud de la France et en Italie, et dans une moindre mesure en Espagne (Albert Martínez Elcacho pour la Catalogne). Les programmes articulant de manière équilibrée recherches de terrain, enquêtes d'archives et analyses archéométriques semblent pour le moment se limiter à ces trois régions et de nombreux espaces riverains de la Méditerranée restent à explorer, soit qu'ils demeurent vierges de recherches (c'est en particulier le cas de l'empire byzantin), soit parce que l'approche s'est limitée à une confrontation des sources écrites et des observations de géologues, parfois assortie de prospections (l'Ifrīqiya abordée par Souha Nefzaoui, la Sardaigne pisane de Jean-Michel Poisson ou la Corse de Florian Leleu et al.). Malgré la grande diversité d'ampleur et de teneur en métaux précieux des gisements, leur exploitation au cours des derniers siècles du Moyen Âge révèle un certain nombre de traits comparables: implication des pouvoirs politiques dans le contrôle voire dans la production, présence d'investisseurs souvent organisés en entreprises, multiplication des règlements miniers, recours fréquent à des techniciens d'origine germanique (qui font de ce domaine un observatoire privilégié des transferts techniques). Si les rythmes de l'activité commencent à être mieux perçus dans quelques espaces (Nicolas Minvielle Larousse pour le Languedoc oriental, Giovanna Bianchi et al., Marco Benvenuti et al. pour les Colline metallifere toscanes, Anna Gattigilia et al. et Lara Casagrande et al. pour le Piémont et le Trentin), le volume de la production demeure partout difficile à estimer et la rentabilité de nombreuses entreprises semble incertaine : les tentatives sans suite et les exploitations ponctuelles ne manquent pas. La contribution de Peter Claughton sur les efforts consentis au $\mathrm{Xv}^{\mathrm{e}}$ siècle pour enrayer le déclin des exploitations minières du Devon apparaît comme déconnectée géographiquement mais elle fournit une illustration supplémentaire du caractère aléatoire de ces investissements.

4 La seconde section invite à suivre quelques étapes de la chaîne opératoire qui va de la mine au demi-produit. Elle débute par des communications en prise directe avec la première partie de l'ouvrage, qui abordent le rôle des différents acteurs de la prospection minière en Bourgogne, Roussillon et Toscane à la fin du Moyen Âge (Joseph Gautier et Catherine Verna, Didier Boisseuil). À nouveau, ces analyses mettent en évidence les risques pris par des investisseurs parfois peu armés pour aborder ce domaine très technique, d'autant que leurs efforts peuvent être spoliés par les pouvoirs princiers dès qu'ils sont couronnés de succès. L'étroite imbrication entre extraction minière, ateliers monétaires et considérations géopolitiques apparaît également dans l'exploitation des filons argentifères du Beaujolais et du Lyonnais (Gérald et Romain Bonnamour). L'étape de la minéralurgie est abordée pour les gisements des $\mathrm{IX}^{\mathrm{e}}$ et $\mathrm{x}^{\mathrm{e}}$ siècles de Melle (Deux-Sèvres) et d'al-Radrad (Yémen), à travers les stratégies d'enrichissement de la galène argentifère en fonction de la teneur en minerai (Florian 
Téreygeol). Plus près des rives de la Méditerranée, l'analyse de la chaîne opératoire de production du cuivre et de ses alliages dans la fonderie de Castel-Minier (Ariège) révèle l'adaptation des artisans de la fin du Moyen Âge à des ressources variées: la chalcopyrite est utilisée pour produire du cuivre non allié alors que le traitement de la bourmonite permet d'élaborer l'alliage ternaire de plomb, de cuivre et d'antimoine alors appelé caldarium (Julie Flament et al.). L'ultime article de cette partie présente le cortège d'analyses géochimiques réalisé dans les Colline metallifere pour mettre en évidence les teneurs en métaux des sols archéologiques et des sédiments des cours d'eau (Vanessa Volpi et al.).

5 La circulation et les usages des métaux précieux constituaient sans doute des thèmes trop vastes pour être abordés dans toute leur diversité. La dernière partie du volume apparaît en conséquence plus pointilliste. Elle débute par une analyse des itinéraires suivis par l'or du Soudan vers al-Andalus à l'époque umayyade (Aurélien Montel) et par un bilan des connaissances sur le rôle de plaque tournante durablement joué par l'oasis de Sijilmassa (Maroc) comme étape de l'or en provenance d'Afrique sahélienne (Chloé Capel). À la fin du xIv e siècle, une partie de l'argent extrait en Bosnie effectue à l'inverse un trajet vers le sud puisque cette matière première est destinée au port d'Alexandrie (Sabine Florence Fabijanec). Le dépouillement d'une ample documentation provençale permet à Olivier Thuaudet de restituer en partie les exportations et importations de cuivre, de plomb et d'étain pour cette région au cours du Moyen Âge tardif. Parmi les sources utilisées à l'occasion de cette enquête, les tarifs de tonlieux semblent constituer un chaînon majeur de notre documentation pour la reconnaissance de la forme, de la dénomination et de la circulation des demi-produits, peu perceptibles dans les sites de production ou de consommation étudiés par l'archéologie. Georges Despy appelait déjà dans les années 1960 à la réalisation de corpus systématiques de ces documents fiscaux mais ce plaidoyer est resté lettre morte.

6 À défaut d'une connaissance matérielle suffisante des étapes qui vont de la production de demi-produits d'or, d'argent ou de cuivre à la réalisation d'objets finis, les analyses archéométriques permettent d'aborder à rebours une partie de ce cheminement en recherchant l'origine du métal utilisé. Une telle démarche est ici proposée à partir des monnaies abbassides du Bilād al-Shām, dont la frappe paraît massivement issue de la refonte de métaux précieux et du commerce, dans une région pauvre en or comme en argent (Cécile Bresc). La fréquence de l'ajout de laiton dans les monnayages d'argent occidentaux des $\mathrm{IX}^{\mathrm{e}}-\mathrm{XI}^{\mathrm{e}}$ siècles constitue un autre acquis récent de la recherche (Guillaume Sarah). L'origine de cet alliage venant altérer l'aloi demeure imprécise et l'hypothèse privilégiée par l'auteur d'une refonte de mobilier en laiton demanderait, pour être validée, la réalisation d'amples campagnes d'analyses de ces objets. Les productions non monétaires en métaux précieux font d'ailleurs une apparition très marginale dans ces actes, sous la forme d'une unique contribution consacrée à l'origine du métal utilisé pour les mosaïques à fond d'or (Elisabetta Neri) : à rebours du possible passage de l'objet au monnayage qui vient d'être évoqué, la refonte de monnaies semble ici privilégiée jusqu'au vi ${ }^{\mathrm{e}}$ siècle, avant que l'approvisionnement en or connaisse une certaine diversification.

7 Les riches apports de ce recueil sont synthétisés par le bilan introductif de MarieChristine Bailly-Maître et par les conclusions de Philippe Braunstein. Le volume révèle aussi des zones d'ombre persistantes pour certaines régions, périodes ou étapes de la production et de l'utilisation des métaux précieux. C'est donc le programme de 
recherches futures qui se dessine en creux. Ainsi, on ne peut que souhaiter l'émergence de programmes pluridisciplinaires dans des espaces ayant jusqu'ici échappé aux recherches de terrain, enquêtes qui considéreraient avec une égale attention la production des différents métaux livrés par les districts étudiés. La possibilité d'une comparaison solidement fondée de l'organisation productive et des techniques en usage à l'échelle du Bassin méditerranéen dépend de cet enrichissement des données. $\mathrm{Au}$ sein du long cheminement qui va de la mine à l'objet recyclé, ce sont probablement les demi-produits issus de la métallurgie qui demeurent aujourd'hui les plus mal appréhendés - y compris dans les rares fonderies étudiées - et il serait utile de s'affranchir des textes traitant spécifiquement des espaces miniers pour analyser les sources fiscales précisant leur origine, leur forme, leur dénomination et leur circulation. À l'étape suivante du processus de production, la nature de l'or, de l'argent et des alliages à base cuivre utilisés pour confectionner d'autres catégories matérielles que les monnaies demeure encore trop mal étudiée. Enfin, évaluer plus précisément le jeu croisé entre refonte des monnaies et refonte des objets nécessitera encore de nombreuses analyses archéométriques.

\section{AUTEURS}

\section{LUC BOURGEOIS}

Centre de recherches archéologiques et historiques anciennes et médiévales (CRAHAM

UMR 6273, CNRS/Université de Caen Normandie) 